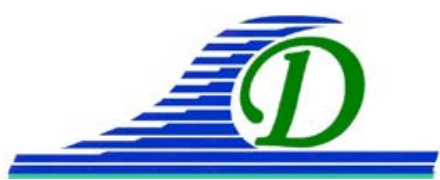

XIII ${ }^{\text {èmes }}$ Journées Nationales Génie Côtier - Génie Civil

Dunkerque, 2-4 juillet 2014

DOI:10.5150/jngcgc.2014.114 ～(C) Editions Paralia CFL

disponible en ligne - http://www.paralia.fr - available online

\title{
Utilisation de la géostatistique pour évaluer des volumes de sédiments pollués
}

\section{Gaël PLASSART ${ }^{1}$, Sara GODOY DEL OLMO ${ }^{1}$}

1. ENVISOL, 37 rue Montgolfier, 38090 Villefontaine France et d'ENVISOL CANADA, 448 place Jacques Cartier, H2Y 3B3 Montréal, Canada. g.plassart@envisol.fr ; s.godoydelolmo@envisol.ca

\section{Résumé :}

Dans le cadre d'un projet de restauration des sédiments présents au sud du quai de Sandy Beach à Gaspé, un diagnostic approfondi des sédiments a été menée de manière systématique dans le but de localiser et de quantifier précisément les déblais à évacuer en filière adaptée dans le cadre de cette décontamination.

Le Ministère de l'Environnement Québécois a souhaité tester un outil d’interpolation différent de ceux utilisés communément au Québec.

Envisol a donc réalisé cette étude géostatistique à l'aide, notamment, de simulations conditionnelles (approche probabiliste) et a pu comparer les différences avec l'approche classique déterministe utilisée couramment au Québec.

Mots clés : Sédiments, Pollution, Cuivre.

\section{Contexte}

Le site à l'étude est une baie en Gaspésie qui servait de débarcadère pour bateau contenant du minerai de cuivre. Lors des opérations de débarquement du minerai celuici s'est déversé au fur et à mesure des années dans la baie, provoquant une contamination des sédiments.

Dans le cadre d'un projet de restauration des sédiments présents au sud du quai de Sandy Beach à Gaspé, une caractérisation détaillée des sédiments a été menée dans le but de cerner la zone d’intervention afin de gérer adéquatement les déblais générés dans le cadre de cette restauration.

Les travaux ont consisté en la réalisation de 129 sondages, dans des secteurs spécifiques bordant la portion sud du quai, dont huit carottes de 0-30 cm dans la zone de marée, 87 carottes de 0-60 cm et 34 carottes de 0-90 cm, dans la zone de dragage prévue. Ainsi, un total de 386 échantillons a été prélevé.

\section{Etude de la contamination par le cuivre}

\subsection{Analyse Exploratoire des données}

Au total, 377 échantillons ont été prélevés dans les sédiments selon un maillage régulier d'environ $25 \times 25$ m, selon une direction régulière nord-ouest/sud-est. 


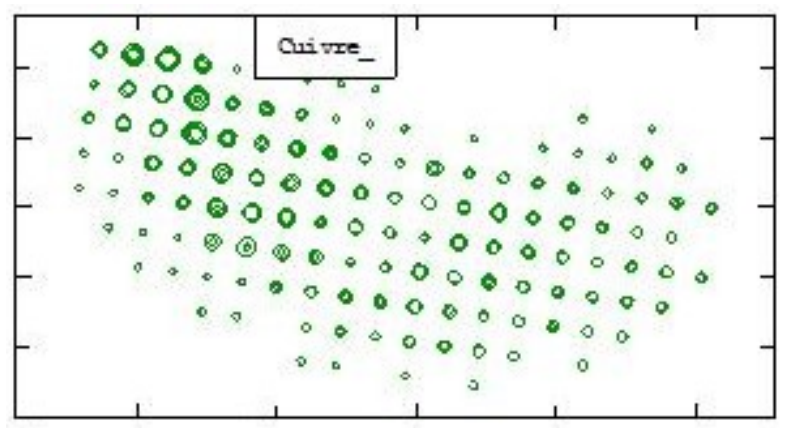

Figure 1. Répartition des prélèvements sur la zone d'étude.

Concernant l'échantillonnage en profondeur, celui-ci a été réalisé tous les $15 \mathrm{~cm}$ jusqu’à une profondeur maximale de $90 \mathrm{~cm}$ environ $(0.00-0.15 \mathrm{~m} ; 0.15-0.30 \mathrm{~m} ; 0.30-0.60 \mathrm{~m}$; 0.60-0.90 cm). Cependant, d'après le graphique ci-dessous (plan XOZ), il apparaît que l'échantillonnage a été réalisé de manière moins importante en profondeur, notamment dans les parties sud et est du site. En effet, dans ces zones, l'échantillonnage a été arrêté à environ $60 \mathrm{~cm}$ de profondeur.

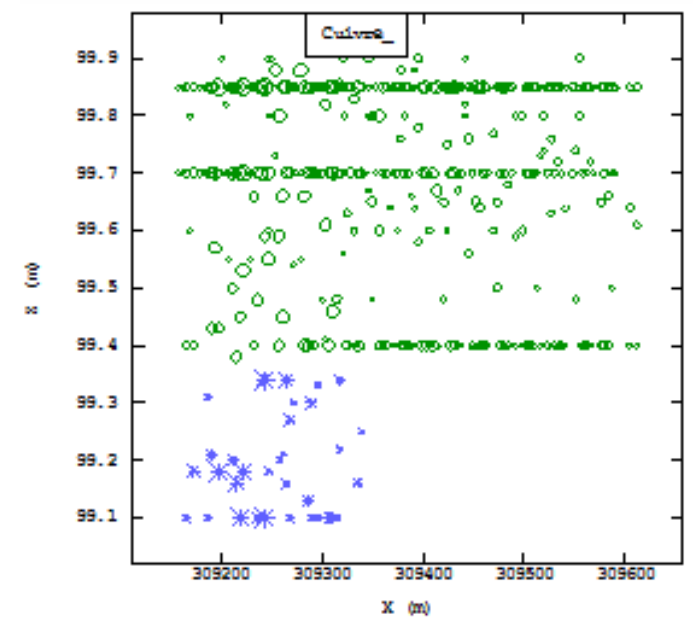

NOTA : la cote des échantillons a été modifiée afin de ne pas obtenir des $Z$ négatifs. La correction a été réalisée comme suit (100-Z d'origine $(\mathrm{cm}))$

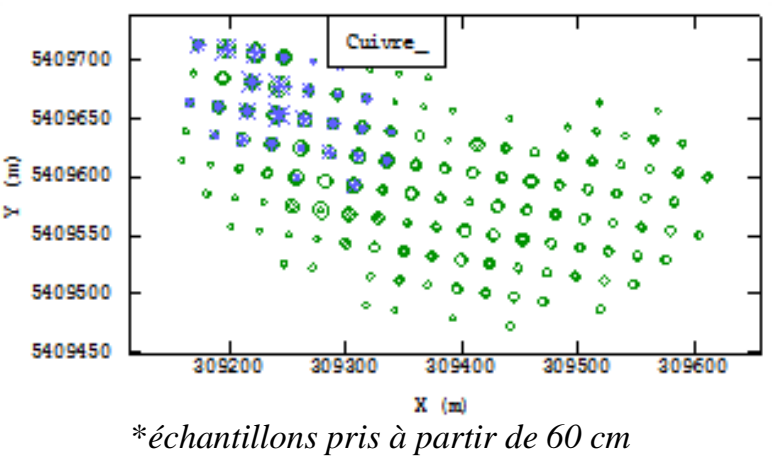

Figure 2. Répartition des prélèvements en fonction de la profondeur.

L'étude de la relation entre profondeur et concentrations en cuivre ne met pas en évidence de corrélation entre la présence d'une contamination et la profondeur de l'échantillon prélevé (coefficient de corrélation de 7.3\%). En effet, des concentrations significatives (supérieures à $2400 \mathrm{mg} / \mathrm{kg}$ ) sont observées sur toute la profondeur investiguée, notamment dans la partie nord-ouest de la zone d'étude. 


\section{XIII ${ }^{\text {èmes }}$ Journées Nationales Génie Côtier - Génie Civil \\ Dunkerque, 2-4 juillet 2014}

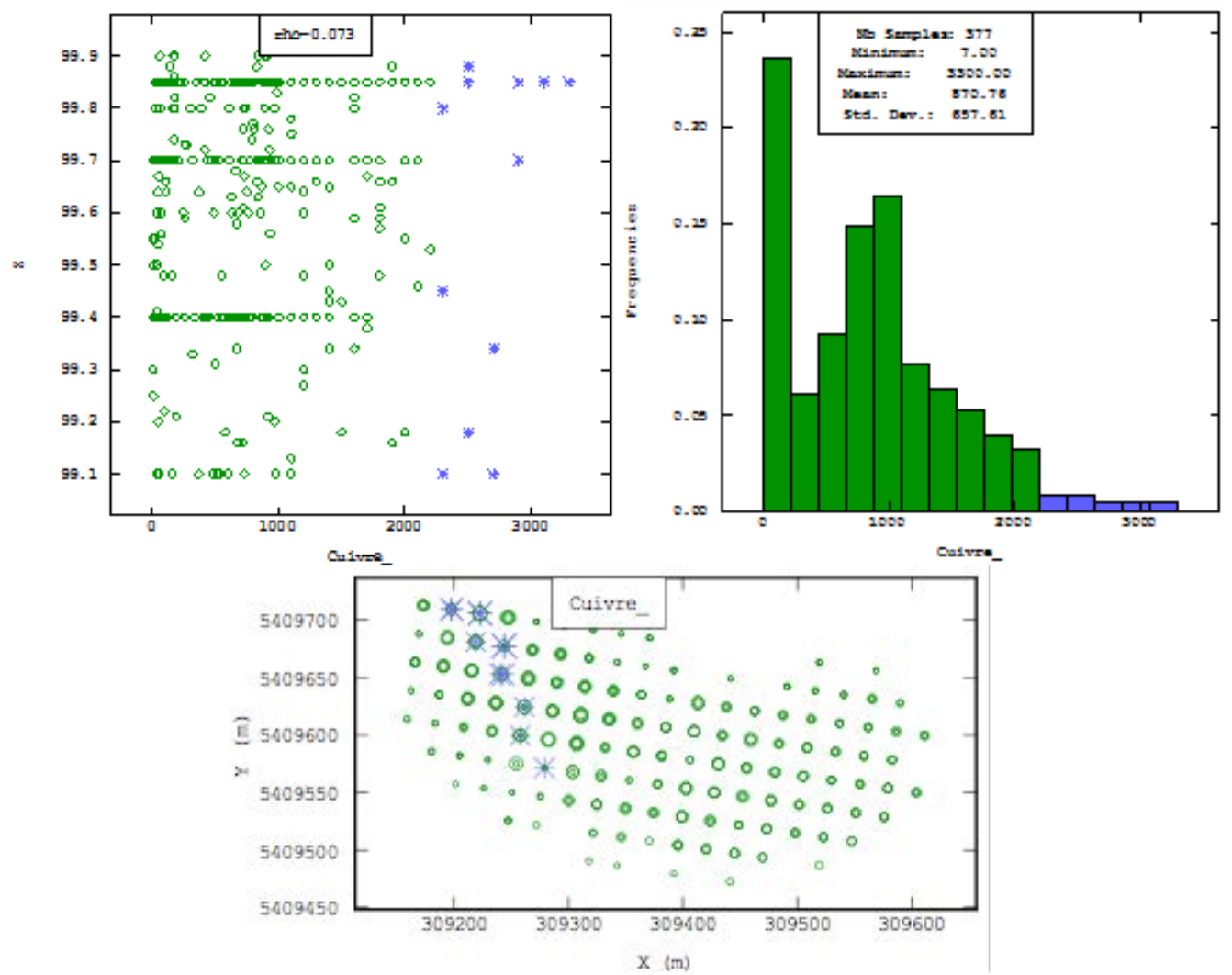

Figure 3. Relation entre la profondeur des échantillons et leurs teneurs en cuivre.

\subsection{Etude des anisotropies}

Le variogramme map réalisé, indique une éventuelle anisotropie selon la direction N110. Pour cette direction, on observe une évolution des concentrations moins significative que pour les autres directions de l'espace. Des variogrammes expérimentaux ont été réalisés en tenant compte de cette anisotropie afin de vérifier la tendance de la pollution selon les différentes directions de l'espace.

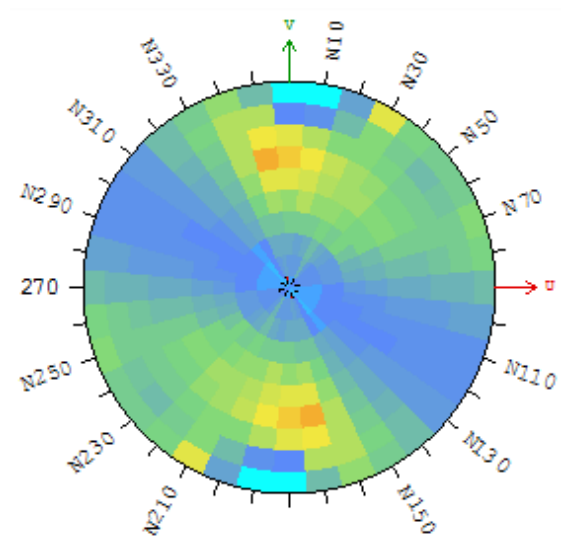

Figure 4. Variogramme map, anisotropie direction N110. 
Compte tenu des premières observations réalisées plusieurs variogrammes sont possibles :

i) Variogramme anisotrope directionnel (NO/D90) selon la direction $110^{\circ}$ par rapport au Nord : ce variogramme tient compte de l'anisotropie identifiée selon les différentes directions de l'espace dans le plan XOY. Aussi, une direction verticale a été prise en compte afin d'étudier la distribution des contaminants selon la profondeur de l'échantillonnage.

ii) Variogramme anisotrope directionnel : la direction verticale n'est pas prise en compte puisque le graphique de corrélation profondeur/concentrations en Cu réalisé précédemment ne montrait pas de corrélation entre ces deux paramètres.

iii)Variogramme isotrope directionnel : ce variogramme ne tient pas compte des anisotropies identifiées dans le plan XOY. En effet, l'anisotropie identifiée selon la direction $\mathrm{N} 110^{\circ}$ correspond également à la direction préférentielle d’échantillonnage.

iv) Variogramme isotrope omnidirectionnel : si on ne tient pas compte des anisotropies ni sur le plan horizontal ni sur la direction verticale.

Les variogrammes obtenus sont présentées dans le rapport RC-SG-1312-002 (GODOY DEL OLMO \& PLASSART, 2013).

\subsection{Modélisation des variogrammes}

Positionnement des données sur le variogramme expérimental - choix des critères :

- portée (zone d'influence) : jusqu'à quelle distance les données estimées sont corrélées ? (on prend généralement 1/3 du champ);

- palier définit par la variance ;

- effet de pépite : définit le comportement à l'origine (faire un choix sur si effet de pépite ou non en réalisant des tests pour voir si cela améliore le modèle) ;

- pente à l'origine : courte ou longue suivant l'évolution spatiale de la contamination (à définir suivant la perception de l’ingénieur vis-à-vis du phénomène).

Seul le cas de de l'anisotrope directionnel (NO/D90) selon la direction préférentielle d'échantillonnage inclinaison de $110^{\circ}$ par rapport au Nord avec effet pépite 0.2 est présentée ci-après. L'ensemble des modélisations est présenté dans le rapport $R C$-SG1312-002 (GODOY DEL OLMO \& PLASSART, 2013). 


\section{XIII ${ }^{\text {èmes }}$ Journées Nationales Génie Côtier - Génie Civil \\ Dunkerque, 2-4 juillet 2014}
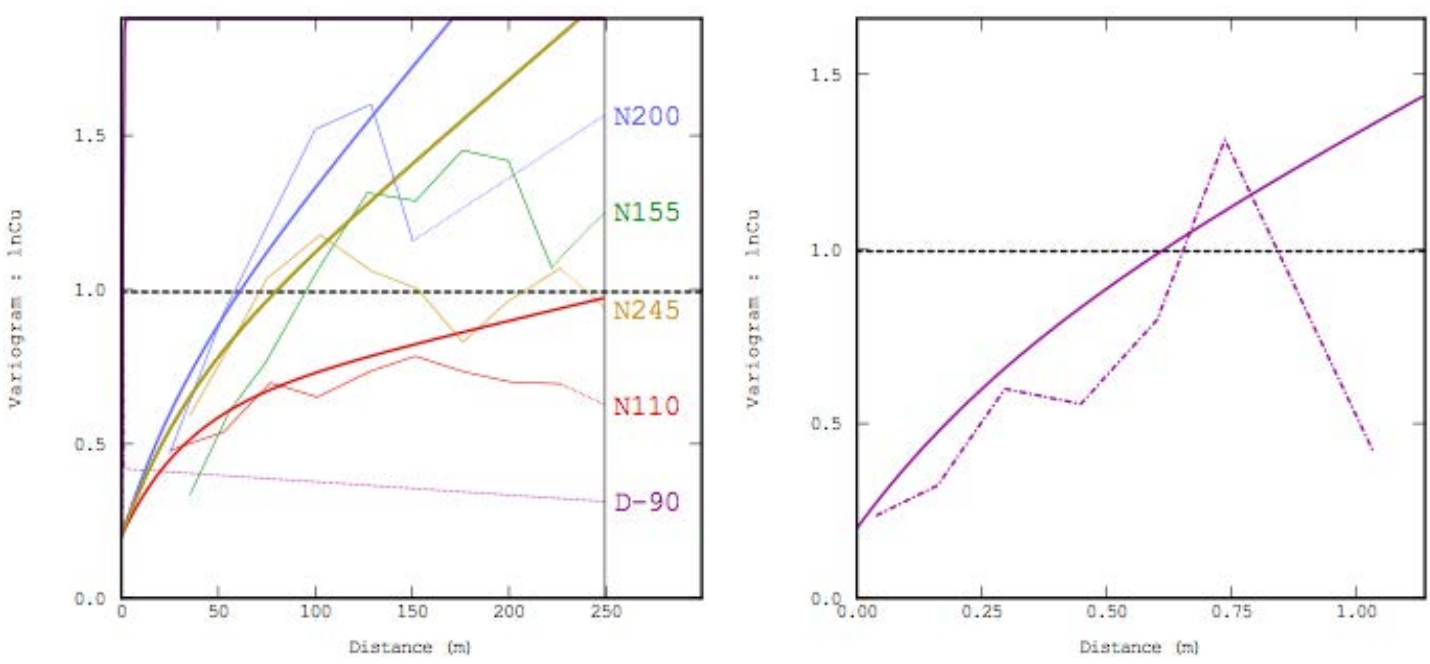

Figure 5. Modèle anisotrope directionnel (NO/D90) selon la direction préférentielle d'échantillonnage inclinaison de $110^{\circ}$ par rapport au Nord avec effet pépite 0.2

\subsubsection{Validations croisées}

Les résultats de la validation croisée sont présentés dans le rapport $R C$-SG-1312-002 (GODOY DEL OLMO \& PLASSART, 2013). Le récapitulatif est détaillé ci-dessous.

Tableau 1. Présentation des différentes performances de variogrammes en fonction des hypothèses émises.

\begin{tabular}{|c|c|c|c|c|c|c|c|c|}
\hline Modèle & $\begin{array}{l}\text { XVAL } \\
\text { Gauss } \\
\end{array}$ & $\begin{array}{l}\text { XVAL } \\
\text { Bbrut }\end{array}$ & $\begin{array}{l}\text { Données } \\
\text { Robustes }\end{array}$ & $\begin{array}{l}\text { Error } \\
\text { Mean }\end{array}$ & $\begin{array}{l}\text { Error } \\
\text { variance }\end{array}$ & $\begin{array}{l}\text { Std error } \\
\text { Mean }\end{array}$ & $\begin{array}{l}\text { Std error } \\
\text { Variance }\end{array}$ & $\begin{array}{l}\text { XVAL } \\
\text { Gauss }\end{array}$ \\
\hline $\begin{array}{l}\text { Anisotrope } \\
\text { (NO-D90) avec EP }\end{array}$ & 0.750 & 0.760 & 370 & -0.01036 & 0.34982 & -0.01462 & 0.67919 & 0.750 \\
\hline $\begin{array}{l}\text { Anisotrope } \\
\text { (NO-D90) sans EP }\end{array}$ & 0.738 & 0.747 & 373 & -0.02542 & 0.39154 & -0.02920 & 0.50593 & 0.738 \\
\hline $\begin{array}{l}\text { Anisotrope } \\
(\mathrm{NO})\end{array}$ & 0.718 & 0.732 & 371 & -0.01333 & 0.39511 & -0.01811 & 0.60920 & 0.718 \\
\hline $\begin{array}{l}\text { Isotrope } \\
\text { NO-D90 }\end{array}$ & 0.762 & 0.771 & 356 & -0.00104 & 0.26511 & -0.00274 & 1.01035 & 0.762 \\
\hline $\begin{array}{l}\text { Omni } \\
\text { avec EP }\end{array}$ & 0.635 & 0.652 & 344 & -0.05991 & 0.29000 & -0.12343 & 1.05749 & 0.635 \\
\hline $\begin{array}{l}\text { Omni } \\
\text { sans EP }\end{array}$ & 0.677 & 0.669 & 134 & 0.01905 & 0.02678 & 0.03953 & 1.88664 & 0.677 \\
\hline
\end{tabular}

\subsubsection{Meilleur modèle isotrope NO-D90}

L'anisotropie identifiée serait plus liée à l'orientation de l'échantillonnage dans une direction préférentielle qu'au comportement de la contamination par le $\mathrm{Cu}$. 


\section{Thème 8 - Gestion et valorisation des sédiments marins}

\subsection{Krigeage des concentrations en Cuivre}

L'estimation des concentrations en un certain polluant peut être obtenue par krigeage à partir des mesures et des modèles de variogrammes précédant.

Le krigeage dépend :

- des distances entre les données et la cible,

- des distances séparant les données entre elles,

- de la structure spatiale du phénomène, étant entendu que pour une même configuration géométrique des données, la cartographie obtenue doit intégrer dans sa construction la continuité spatiale du phénomène (par exemple très lisse ou au contraire hétérogène).

La configuration des données, la position du point où l'on veut réaliser l'estimation et le modèle de variogramme étant connus, le krigeage consiste à déterminer les poids de krigeage, seules inconnues d'un système d'équations qu'il faut résoudre. Ces poids permettront de :

- garantir le non-biais de l'estimation,

- minimiser la variance de l'erreur d'estimation.

En ce sens, le krigeage constitue le meilleur estimateur linéaire sans biais.

En pratique, l'estimation en un point donné peut être réalisée à partir de tous les points de mesure disponibles (voisinage unique) ou uniquement à partir des points situés dans un certain voisinage du point cible (voisinage glissant).

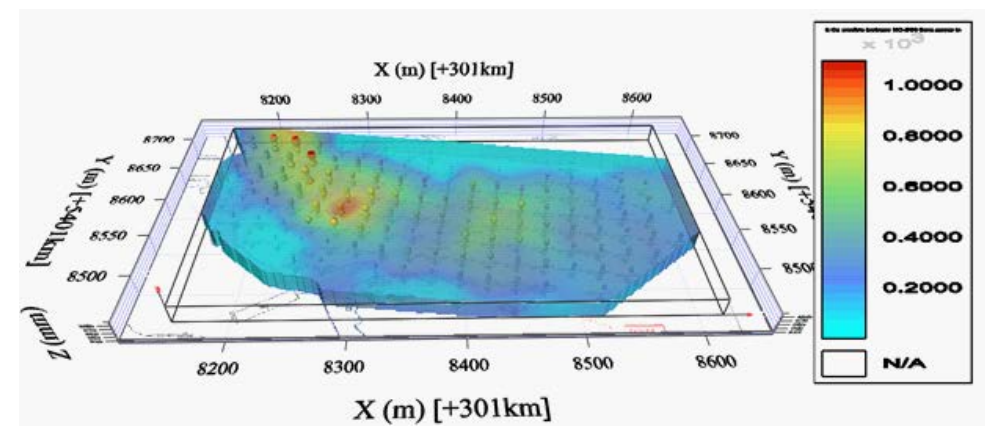

Figure 6. Représentation 3D du modèle isotrope NO-D90.

\subsection{Simulations conditionnelles}

L'estimation des volumes dépassant un objectif de réhabilitation (volumes dits "contaminés") doit répondre à deux questions :

- quelles sont les zones contaminées ? Cette question permet d'estimer localement le risque de dépassement d'un objectif de réhabilitation.

- quel est le volume contaminé ? Cette question permet d'estimer globalement le niveau de contamination de la zone d'étude.

Le volume contaminé va dépendre de la répartition spatiale des concentrations mesurées. Une cartographie par krigeage des concentrations n'est pas suffisante en vue 


\section{XIII ${ }^{\text {èmes }}$ Journées Nationales Génie Côtier - Génie Civil \\ Dunkerque, 2-4 juillet 2014}

d'un calcul de volume contaminé, car elle néglige l'incertitude liée à l'estimation du phénomène en tout point de la zone d'étude à partir de quelques échantillons. Ainsi, afin de pouvoir caractériser cette incertitude, une approche par simulations géostatistiques est préconisée. Chaque simulation correspond à un scénario possible pour la répartition des concentrations, qui respecte :

- les valeurs observées aux points de mesures,

- la distribution statistique de ces valeurs sur la zone d'étude,

- la continuité spatiale des concentrations, appréhendée par le variogramme des mesures.

Les cartes dans le rapport $R C-S G$-1312-002 (GODOY DEL OLMO \& PLASSART, 2013), montrent une contamination par le $\mathrm{Cu}(2400 \mathrm{mg} / \mathrm{kg})$, notamment dans la partie nord-ouest du site et suivant une direction nord-ouest/sud-est.

\section{Etude de la contamination par les HAP}

Le processus géostatistique a été repris afin d'étudier les concentrations en HAP dans la zone d'étude.

\subsection{Analyse exploratoire des données}

Total de 369 (maillage $25 \times 25 \mathrm{~m}$ ) jusqu'à $90 \mathrm{~cm}$ de profondeur. Etude par rapport au seuil fédéral de $5 \mathrm{mg} / \mathrm{kg}$ pour la somme des HAP.

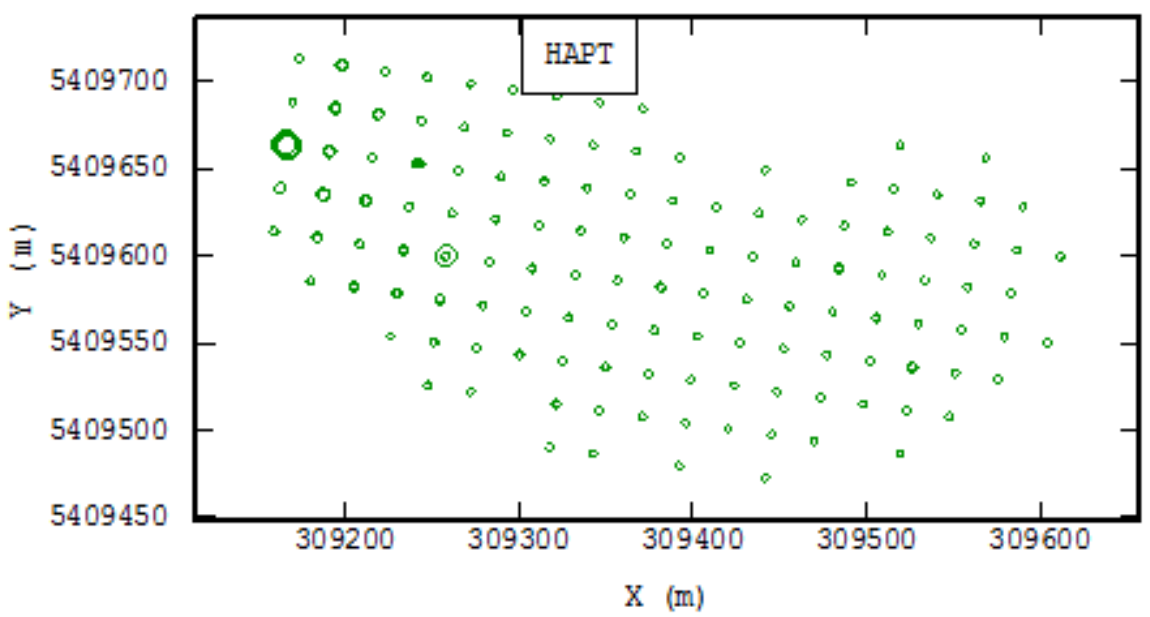

Figure 7. Représentation des prélèvements sur la zone d'étude.

L’analyse exploratoire détaillée dans le rapport $R C$-SG-1312-002, met en évidence les points suivants :

- des teneurs supérieures au seuil fédéral de 5 mg/kg sur majeure partie du site ;

- sur toute la profondeur investiguée ; 
- des échantillons ont été prélevés en profondeur à partir de $60 \mathrm{~cm}$ uniquement dans la partie nord-ouest du site.

Les variogrammes expérimentaux sont également présentés dans le rapport $R C$-SG1312-002.

\subsection{Modélisation du variogramme}

La validation croisée est détaillée dans le rapport $R C$-SG-1312-002 (GODOY DEL OLMO \& PLASSART, 2013).
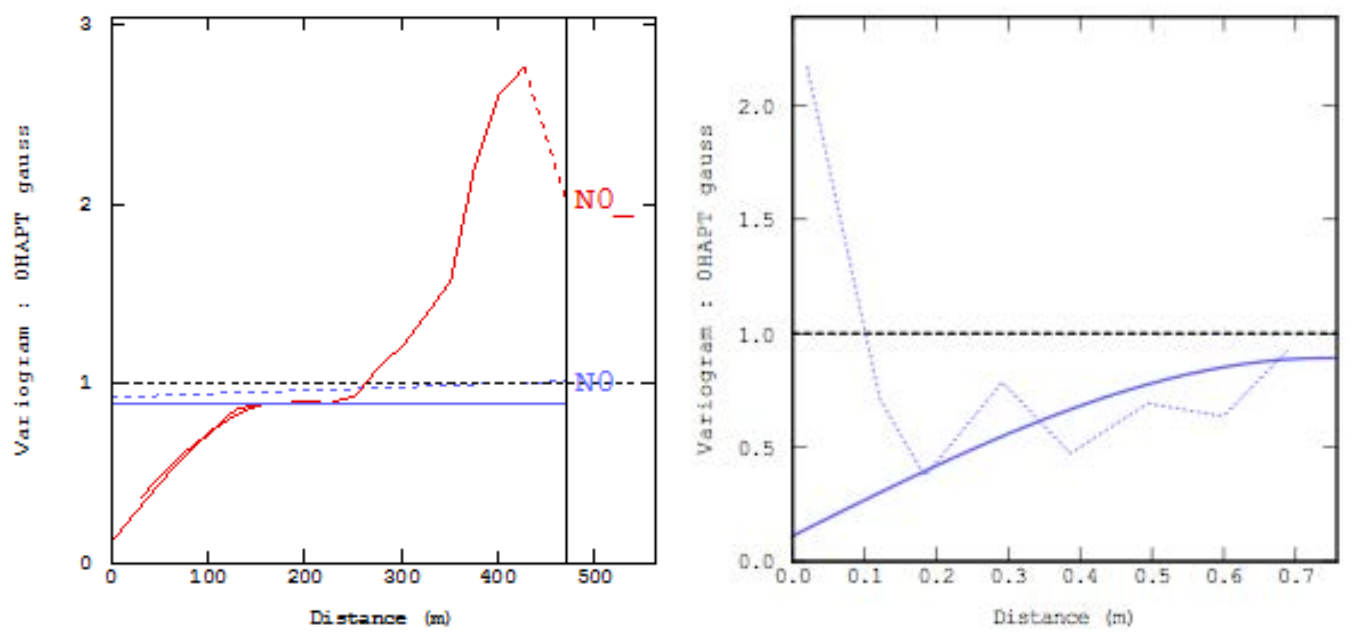

Figure 8. Variogrammes expérimentaux de la concentration en HAP selon 2 directions.

\subsection{Krigeage}

Partie détaillée dans le rapport RC-SG-1312-002 (GODOY DEL OLMO \& PLASSART, 2013).

\subsection{Simulations conditionnelles}

L'étude des concentrations en HAP montrent les éléments suivants :

- un degré important de contamination mise en évidence sur les échantillons prélevés aussi bien en surface qu'en profondeur, et

- une densité d'échantillonnage moins importante en profondeur, notamment dans la partie sud et est du site,

Cela se traduit par la présence de sédiments montrant une incertitude de l'estimation, notamment dans les couches profondes, dans les parties où la densité d'échantillonnage est moins importante.

Les simulations géostatistiques réalisées sur un support de $5 \times 5 \times 0.15 \mathrm{~m}$ montrent qu'une probabilité de $26 \%$ de dépasser le seuil fédéral de 5 ppm, nous permettrai de cartographier l'ensemble des zones dépassant ce critère mais met en évidence un 


\section{XIII ${ }^{\text {èmes }}$ Journées Nationales Génie Côtier - Génie Civil \\ Dunkerque, 2-4 juillet 2014}

volume important de sédiments présentant de l'incertitude en profondeur, notamment sur les parties est et sud du site.

La courbe de risque issue des simulations géostatistiques montre un volume probable de $43200 \mathrm{~m}^{3}$ correspondant à une faible probabilité de dépasser le critère retenu (5 mg/kg). Sur ce volume, un total de $14500 \mathrm{~m}^{3}$ correspond à des blocs montrant de l'incertitude (entre 30 et $60 \%$ de dépasser le seuil de 5 ppm), soit 34\% du volume total annoncé.

Compte tenu de la prise en compte de ces incertitudes, le résultat annoncé par la méthode géostatistique est 2 fois plus important que celui annoncé lors des études précédentes réalisées sur la zone.

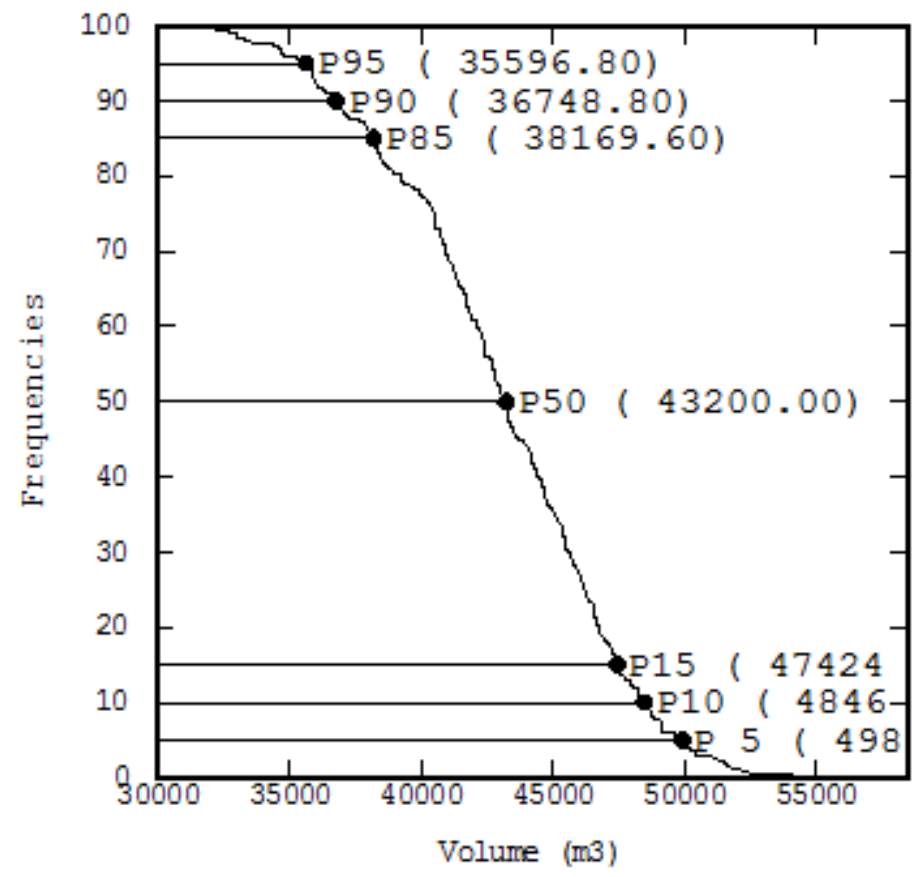

Figure 9. Courbe de risque issue des simulations géostatistiques

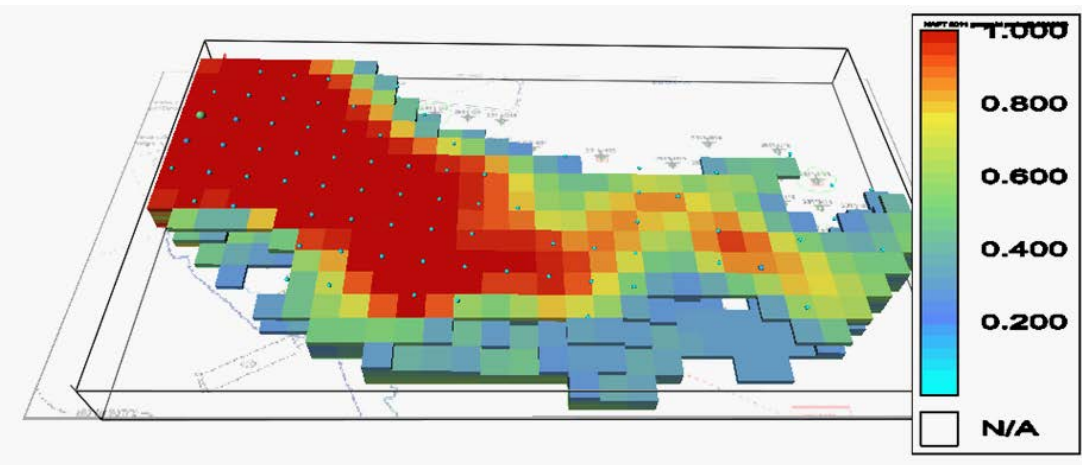

zones de forte incertitude, pas de donnée en profondeur

Figure 10. Probabilité de dépassement des seuils en HAP issue des simulations géostatistiques 


\section{Conclusions}

L'étude géostatistique menée sur les sédiments de la baie Sandy Beach de Gaspé au Canada a permis de mettre en évidence la structure spatiale des contaminations en Cuivre et HAP.

Il a alors été possible de déterminer finement les volumes contaminés grâce à une approche probabiliste par simulations géostatistiques conditionnelles et surtout de quantifier les incertitudes liées à ces volumes.

Cette approche se distingue donc des autres méthodes d'interpolation déterministes qui ne prennent pas en compte la structure spatiale de la pollution et ne peuvent pas quantifier les incertitudes liées au calcul de volume. Les études géostatistiques sont donc un formidable outil d'aide à la décision pour un maître d'ouvrage.

Cette étude a aussi pu mettre en évidence que le plan d'échantillonnage peut influencer négativement les calculs de volume de terres contaminées en masquant les structures spatiales.

Il serait intéressant de tester d'autres types de plan d'échantillonnage afin de vérifier leur adéquation avec les objectifs fixés (par exemple un échantillonnage systématique aléatoire).

\section{Référence bibliographique}

GODOY DEL OLMO S., PLASSART G. (2013). Etude géostatistique sur la répartition tridimensionnelle des contaminants cuivre et HAP dans la baie de Gaspé Sandy Beach. Réf. : RC-SG-1312-002. 